\section{High-performance ZnO thin-film transistors fabricated at low temperature on glass substrates}

\section{C.C. Liu, Y.S. Chen and J.J. Huang}

A high-performance enhancement-mode $\mathrm{ZnO}$ thin-film transistor (TFT) on a glass substrate is demonstrated. The $\mathrm{ZnO}$ thin film is deposited by RF magnetron sputtering with the presence of $\mathrm{O}_{2}$ at low deposition rate and low temperature. The $I_{D S}$ is as high as $1 \mathrm{~mA}$ when biased at the saturation region $V_{D S}=10-20 \mathrm{~V}$ and $V_{G S}=5 \mathrm{~V}$ without any post-thermal anneal. The $I_{\mathrm{on}} / I_{\text {off }}$ ratio is $3 \times 10^{6}$. The results are among the best $\mathrm{ZnO}$ TFTs ever obtained.

Introduction: The wide bandgap $\mathrm{ZnO}$ semiconductor material has attracted much attention recently for applications in transparent circuits and on flexible substrates for flat panel displays. The invisible transistors on thin-film transistor-liquid crystal displays (TFT-LCDs) have the advantage of a higher aperture ratio for the transmission of backlight. Several approaches have been used to deposit $\mathrm{ZnO}$ thin films, including sputtering [1], pulsed laser deposition [2], chemical vapour deposition [3] and molecular beam epitaxy [4]. Most of these methods require high temperature process during $\mathrm{ZnO}$ coating and contact alloy in order to obtain better crystalline quality and contact resistance. To apply $\mathrm{ZnO}$ to flat panel displays, especially on flexible substrates, low-temperature deposition was also proposed by sputtering, spin-coating, and chemical bath [5-7]. The device performance, depending on the process conditions and material properties such as crystalline quality (and thus the carrier mobility) in the $\mathrm{ZnO}$ channel, from the above approaches typically showed an $I_{\text {on }} / I_{\text {off }}$ ratio around $10^{5}$ to $10^{6}$ and $I_{D S}$ around 6 to $60 \mu \mathrm{A}$ when biased at $V_{D S}$ and $V_{G S}$ around 30-40 V [6-9]. To apply ZnO TFTs to active matrix LCDs (AMLCDs), higher operating currents at lower bias voltages are required.

In this Letter, we demonstrate a high-performance enhancementmode $\mathrm{ZnO}$ TFT on a glass substrate. We utilised a top-gate approach to fabricate the devices. The $\mathrm{ZnO}$ thin film is deposited by $\mathrm{RF}$ magnetron sputtering at low deposition rate and low temperature with the presence of $\mathrm{O}_{2}$. The $I_{D S}-V_{D S}$ curves, $I_{\mathrm{on}} / I_{\mathrm{off}}$ ratio and leakage current $I_{G S}$ are compared before and after contact alloy. We believe the results are among the best ZnO TFTs ever obtained.

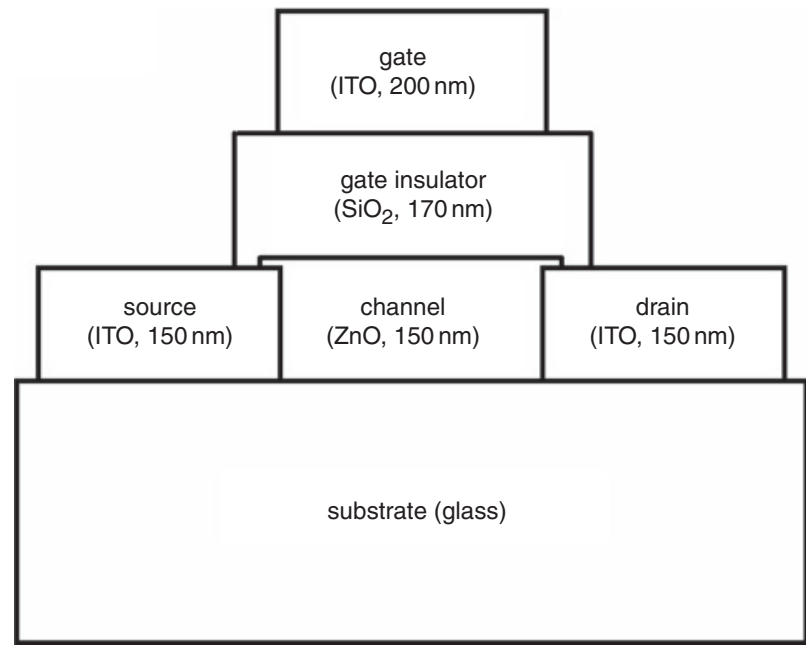

Fig. 1 Schematic cross-sectional view of TFT structure

Experiment: Fig. 1 schematically illustrates a top-gate-type transparent TFT device using $\mathrm{ZnO}$ as the active channel layer. First, a glass substrate (Corning 1737 glass) is blanket-sputtered with a highly transparent, $n$-type indium tin oxide (ITO). The ITO layer thickness is $150 \mathrm{~nm}$. The ITO source and drain regions are then defined by photolithography and wet etching $\left(\mathrm{HCl}: \mathrm{H}_{2} \mathrm{O}_{2}=1: 3\right)$. We then deposited the $150 \mathrm{~nm}$-thick $\mathrm{ZnO}$ channel layer by $\mathrm{RF}$ magnetron sputtering in $10^{-2}$ torr of $\mathrm{Ar} / \mathrm{O}_{2}(15: 1)$. The purpose of oxygen gas is to decrease the carrier density of the $\mathrm{ZnO}$ layer so that enhancementmode TFTs can be obtained. During deposition, RF power was $100 \mathrm{~W}$ and the wafer holder was attached to a water running pipe to remove the generated heat. Moreover, the deposition rate was kept at around
$5 \mathrm{~nm} / \mathrm{min}$ to mitigate the particle bombardment to achieve better polycrystalline quality. The channel width and length were 1000 and $300 \mu \mathrm{m}$, respectively, yielding a width-to-length ratio 3.3. In the subsequent step, a $170 \mathrm{~nm}$-thick $\mathrm{SiO}_{2}$, layer was sputtered as the gate insulator. Again, we adapted a slow deposition rate approach $(2 \mathrm{~nm} / \mathrm{min}$ in this work) with $100 \mathrm{~W} \mathrm{RF}$ power. Finally, the gate electrode region was coated with a $200 \mathrm{~nm}$ sputtered ITO layer. All ITO electrodes, including source, drain and gate contacts, were sputtered using $120 \mathrm{~W}$ RF power with higher deposition rate $20 \mathrm{~nm} / \mathrm{min}$.

Results and discussion: The current-voltage characteristics were measured using an Agilent $4155 \mathrm{C}$. Measurements were performed in darkness to avoid light induced photocurrents. The $I_{D S}-V_{D S}$ curves of the ZnO TFT before contact alloy are shown in Fig. $2 a$. The $I_{D S}$ is $1.06 \mathrm{~mA}$ at the bias condition $V_{D S}=5 \mathrm{~V}$ and $V_{D S}=20 \mathrm{~V}$. Our enhancement-mode $\mathrm{ZnO}$ thin-film transistor implies that the power dissipation can be minimised when the device is normally-off, making it suitable for applications about which power consumption is a concern. Fig. $2 b$ shows the transfer characteristics and gate leakage current. The $I_{\mathrm{on}} / I_{\mathrm{off}}$ ratio, calculated from the drain current at its maximum and minimum value, is $3 \times 10^{6}$. The gate leakage current is smaller than $3 \times 10^{-8} \mathrm{~A}$ with $V_{G S}$ less than $7 \mathrm{~V}$, which is attributed to a high-quality $\mathrm{SiO}_{2}$ gate insulting layer during device fabrication. Furthermore, the threshold voltage, determined from the $\left(I_{D S}\right)^{1 / 2}-V_{G S}$ curve, is around $1.7 \mathrm{~V}$. The low threshold voltage ensures that $\mathrm{ZnO}$ TFT circuits are operated at low voltage and thus low power consumption can be achieved.
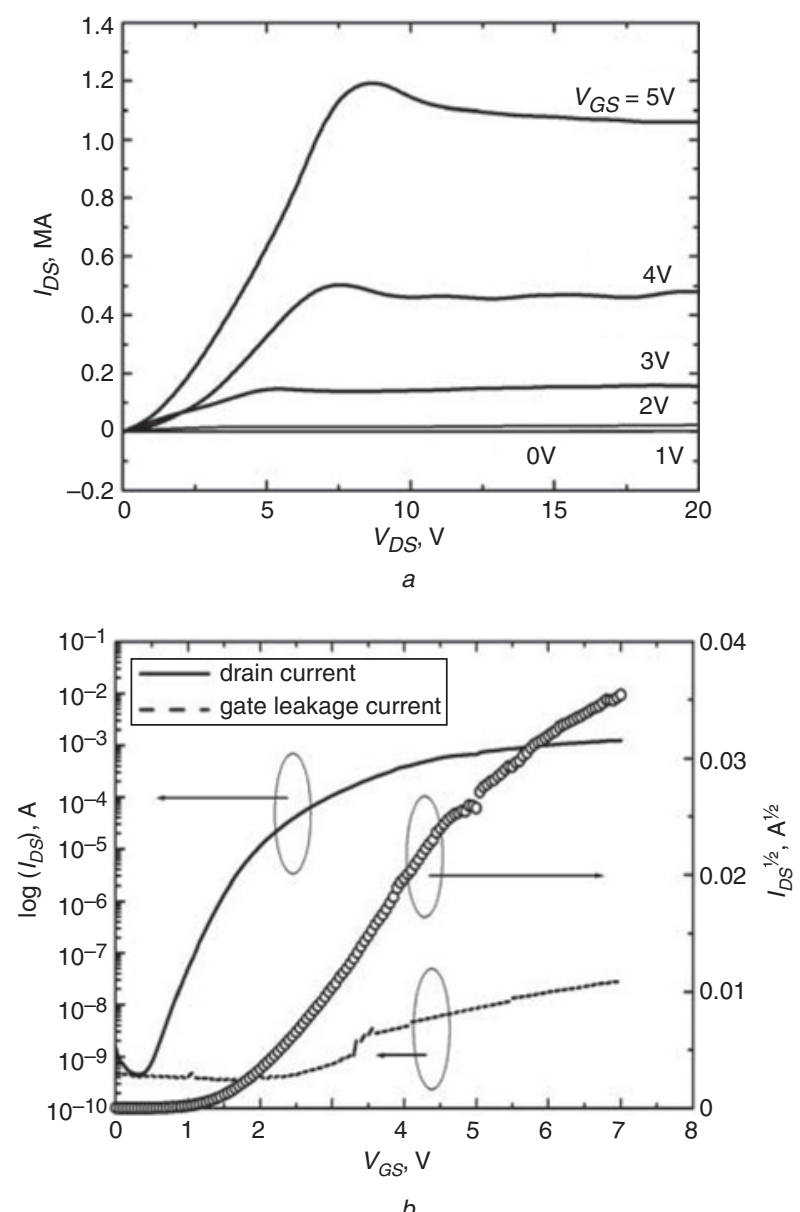

Fig. 2 Electrical characteristics of TFT with $\mathrm{ZnO}$ channel and $\mathrm{SiO}_{2}$ gate insulator before alloy

a $I_{D S}-V_{D S}$ curves

$b$ Log scale $I_{D S}$ and $I_{G S}$ against $V_{G S}$ at $V_{D S}=20 \mathrm{~V}$, and $\left(I_{D S}\right)^{1 / 2}-V_{G S}$ curve for determining threshold voltage

It is observed that a high contact resistance between the source/drain electrode and the $\mathrm{ZnO}$ channel layer in the triode region and an overshoot at the onset of saturation exist in Fig. $2 a$. The $I_{D S}-V_{D S}$ 
curves behave like a TFT device in series with a Schottky diode component. To decrease contact resistance the sample is then subject to a $200^{\circ} \mathrm{C}, 15 \mathrm{~min}$ rapid thermal process in $\mathrm{N}_{2}$. The electrical characteristics are shown in Fig. 3. The currents in the saturation region in Fig. $3 a$ show little change from those before alloy, indicating that the carrier mobility in the $\mathrm{ZnO}$ layer is not affected by the alloy condition. The slopes of $I_{D S} V_{D S}$ curves in the triode region are much more linear and the overshoot at the onset of the saturation region is reduced for devices after alloy. We attribute the improvement of device performance to a better contact condition between the source/drain ITO and the $\mathrm{ZnO}$ layer. As for the transfer characteristics, the $I_{\text {on }} / I_{\text {off }}$ ratio becomes $3 \times 10^{4}$ and the gate leakage current increases to $2 \times 10^{-7} \mathrm{~A}$ at $V_{G S}=7 \mathrm{~V}$ after thermal anneal.
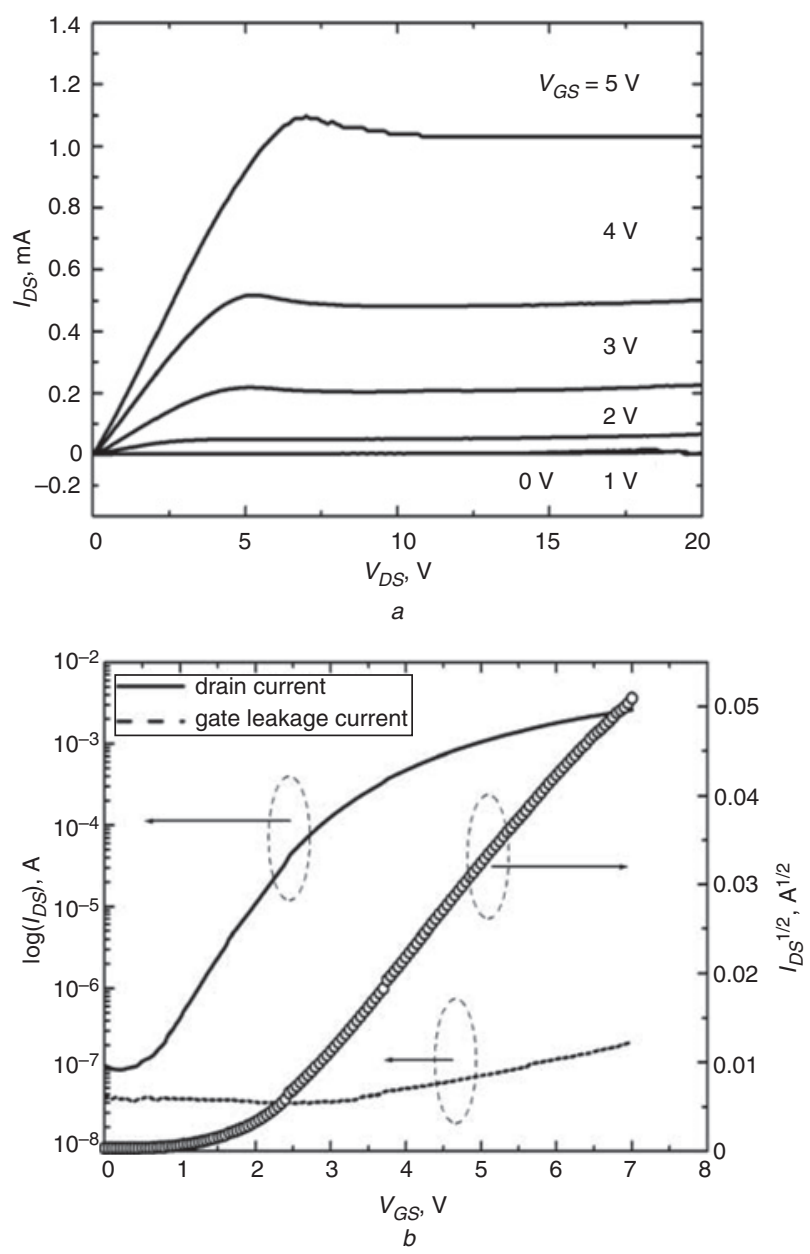

Fig. 3 Electrical characteristics of TFTs subject to $200^{\circ} \mathrm{C}, 5 \mathrm{~min}$ rapid thermal process in $\mathrm{N}_{2}$

a $I_{D S} V_{D S}$ curves

$b$ Log scale $I_{D S}$ and $I_{G S}$ against $V_{G S}$ at $V_{D S}=20 \mathrm{~V}$, and $\left(I_{D S}\right)^{1 / 2}-V_{G S}$ curve
Conclusion: We have demonstrated a high-performance enhancementmode ZnO TFT on a glass substrate. Before contact alloy, the $I_{D S}$ is as high as $1 \mathrm{~mA}$ when biased at the saturation region $V_{D S}=10-20 \mathrm{~V}$ and $V_{G S}=5 \mathrm{~V}$. The $I_{\mathrm{on}} / I_{\text {off }}$ ratio is $3 \times 10^{6}$. The source and drain contacts are improved after $200^{\circ} \mathrm{C}, 15 \mathrm{~min}$ alloy. The slopes of $I_{D S}-V_{D S}$ curves in the triode region are much more linear and the overshoot at the onset of saturation region is reduced. The fabrication steps developed and the $\mathrm{ZnO}$ TFT performance in this work are suitable for AMLCD applications.

(C) The Institution of Engineering and Technology 2006

16 May 2006

Electronics Letters online no: 20061518

doi: 10.1049/el:20061518

C.C. Liu, Y.S. Chen and J.J. Huang (Graduate Institute of Electro-Optical Engineering, National Taiwan University, 1 Roosevelt Rd, Sec.4, Taipei 106, Taiwan, Republic of China)

E-mail: jjhuang@cc.ee.ntu.edu.tw

\section{References}

1 Yao, Q.J., and Li, D.J.: 'Fabrication and property study of thin film transistor using rf sputtered $\mathrm{ZnO}$ as channel layer', J. Non-Cryst. Solids, 2005, 351, pp. 3191-3194

2 Kim, I.-D., Choi, Y.W., and Tuller, H.L.: 'Low-voltage $\mathrm{ZnO}$ thin-film transistors with high- $K \mathrm{Bi}_{1.5} \mathrm{Zn}_{1.0} \mathrm{Nb}_{1.5} \mathrm{O}_{7}$ gate insulator for transparent and flexible electronics', Appl. Phys. Lett., 2005, 87, p. 043509

3 Minegishi, K., Koiwai, Y., Kikuchi, Y., Yano, K., Kasuga, M., and Shimizu, A.: 'Growth of $p$-type zinc oxide film by chemical vapor deposition', J. Appl. Phys., Part 2, 1997, 36, p. 1453

4 Tang, Z.K., Wong, G.K.L., Yu, P., Kawasaki, M., Ohtomo, A., Koinuma, H., and Segawa, Y.: 'Room-temperature ultraviolet laser emission from selfassembled ZnO microcrystallite thin films', Appl. Phys. Lett., 1998, 72, pp. 3270-3272

5 Fortunato, E.M.C., Barquinha, P.M.C., Pimentel, A.C.M.B.G., Conçalves, A.M.F., and Martins, R.F.P.: 'Fully tranparent $\mathrm{ZnO}$ thin film transistor produced at room temperature', Adv. Mater., 2005, (5), pp. 590-594

6 Norris, B.J., Anderson, J., Wager, J.F., and Keszler, D.A.: 'Spin-coated zinc oxide transparent transistors', J. Phys. D, Appl. Phys., 2003, 36, pp. L105-L107

7 Cheng, H.-C., Chen, C.-F., and Lee, C.-C.: 'Thin-film transistors with active layers of zinc oxide $(\mathrm{ZnO})$ fabricated by low-temperature chemical bath method', Thin Solid Films, 2006, 498, pp. 142-145

8 Carcia, P.F., McLean, R.S., Reilly, M.H., and Nunes, G., Jr.,: 'Transparent $\mathrm{ZnO}$ thin-film transistor fabricated by rf magnetron sputtering', Appl. Phys. Lett., 2002, (7), pp. 1117-1119

9 Hoffman, R.L., Norris, B.J., and Wager, J.F.: 'ZnO-based transparent thin-film transistors', Appl. Phys. Lett., 2003, 82, (5), pp. 733-735 